\title{
Long - Term Effects of Topical Amniotic Membrane Stem Cell Metabolite Product (AMSC-MP) and Fractional CO2 Laser in Photoaging
}

\author{
Dyah Ayu Pitasari, Afif Nurul Hidayati, Sawitri, Damayanti, Cita Rosita Sigit \\ Prakoeswa, Muhammad Yulianto Listiawan. \\ Departement of Dermatology and Venereology \\ Faculty of Medicine Universitas Airlangga/Dr. Soetomo General Academic Teaching Hospital \\ Surabaya
}

\begin{abstract}
Background: Photoaging causes wrinkles, lentigines, keratoses, dyspigmentation, telangiectasia, decreased elasticity, rough texture, and pale color. Facial wrinkles and mottled skin can negatively affect perceived attractiveness, self-esteem, personality, and level of acceptance. Stem cells are important cells in the skin, and they are the source for continuous regeneration of the epidermis. It can be extracted from many sources, including amnion. Amnion membrane stem cell (AMSC) can be cultured and secretes metabolite product, and it can rejuvenate aging skin. Laser-Assisted Drug Delivery (LADD) involves selective destruction of the layers of the epidermis and dermis to allow penetration and absorption of topical drugs as well as large molecular weight drugs such as stem cells. Purpose: To evaluate the long-term effects of topical administration of AMSC metabolite products (AMSC -MP) and fractional $\mathrm{CO}_{2}$ laser on the clinical improvement of photoaging compared to previous skin conditions. Methods: This was an observational study which was aimed to evaluate the long-term effects of clinical improvement after topical therapy of AMSC-MP and fractional $\mathrm{CO}_{2}$ laser in patients with photoaging. Results: The results of the analysis showed a decrease in wrinkles, dark spots, skin tone, and pores. Conclusion: Based on Janus-II ${ }^{\circledR}$ assessment, there was improvement degrees of wrinkles, dark spots, and pores in patients with photoaging who received fractional $\mathrm{CO}_{2}$ laser fractional for 12 weeks. There were no side effects found in this study.
\end{abstract}

Key words: Amniotic membrane, stem cell, fractional $\mathrm{CO}_{2}$ laser, photoaging.

Correspondence address: Muhammad Yulianto Listiawan, Department of Dermatology and Venereology, Faculty of Medicine, Universitas Airlangga, Dr. Soetomo General Academic Teaching Hospital, Jl. Prof. Dr. Moestopo street No. 6-8 Surabaya 60131, Indonesia. Telephone: +6231-5501609, e-mail: yuliantowawan@yahoo.com.

\section{INTRODUCTION}

Photoaging is a complex biological process that is influenced by a combination of intrinsic and extrinsic factors but is more dominated by extrinsic factors such as chronic light exposure, pollution, ionizing radiation, chemicals, and toxins. The most common source of environmental damage is ultraviolet (UV) radiation. The degree of sun damage is directly proportional to the amount of exposure and inversely proportional to the amount of genetically determined skin pigmentation. Damage caused by solar UV radiation is called photoaging and superimposed on chronological aging. ${ }^{1}$ Photoaging produces wrinkles, lentigines, keratosis, dyspigmentation, telangiectasia, decreased elasticity, rough texture, and pale color. ${ }^{2}$ Facial wrinkles and mottled skin can negatively affect perceived attractiveness, self-esteem, personality, and level of acceptance. The appearance of aging skin, especially in women, can cause mood disorders and decreased self-confidence; thus, it affects the quality of life. ${ }^{3}$ Indonesia is a tropical country with all-year sun exposure, making Indonesians very vulnerable to photoaging. ${ }^{4}$

Stem cells are important cells in the skin because they are the source of continuous regeneration of the epidermis. Stem cells have the potential for selfrenewal and differentiation. Stem cell therapy has prevented disease and tissue repair. Human stem cells release various growth factors, cytokines, and other extracellular matrix (ECM) regulating agents, including type 1 and 3 collagen, elastin, and fibronectin in Human Dermal Fibroblasts (HDF). ${ }^{5}$ Stem cells refer to various types of cells which are not specific; cells that are able to produce one or more types of cell lines from the germinal layer and have the ability to renew themselves. The human placenta, which is routinely discharged postpartum, irrespective of its natural aging process, is still a rich source of stem cells capable of proliferating and in vitro differentiating in many directions. In addition to homing and differentiation in the area of injury, mesenchymal stem cells have a strong paracrine effect, which stimulates the repair process. ${ }^{6}$ Laser-Assisted Drug Delivery (LADD) involves selective destruction of the layers of 
epidermis and dermis to allow penetration and absorption of topical drugs as well as large molecular weight drugs. LADD is fractionally ablative, and it functions by creating a focused damage zone to selective episodes so that the dermis is more receptive to topical medications. ${ }^{7}$ The efficacy of topical therapy depends on the ability of the therapeutic drug to achieve its targets. Large lipophilic and hydrophilic drugs cannot penetrate normal intact skin. It is expected that as the penetration reaching the target, the therapy will have more prolonged effect, and it is promising as a photoaging therapy option in the future. ${ }^{7}$

According to research by Prakoeswa and colleagues, micro-needling and AMSC- MP demonstrated a significant percentage of improvement in photoaging treatment compared to microneedling and normal saline. This study showed clinical improvement of pores and other photoaging manifestation at eight weeks. Lee and Colleagues studied adipocyte-derived stem cell-secreted products for up to 12 weeks. ${ }^{8}$ This study was conducted to assess the long-term effects of topical administration of AMSC-MP and fractional $\mathrm{CO}_{2}$ laser on the clinical improvement of photoaging by using the Facial Analysis System Janus-II ${ }^{\circledR}$.

\section{METHODS}

This was an observational study evaluating the long-term effects of clinical improvement of topical of AMSC-MP after fractional $\mathrm{CO}_{2}$ laser in photoaging. This study used a pair of samples in accordance with the inclusion and exclusion sample criteria that consisted of 30 objects. Inclusion criteria were women with a degree of photoaging II-III based Glogau, aged 40-65 years, received AMSC-MP and fractional laser $\mathrm{CO}_{2}$ every 4 weeks for 12 weeks and signed informed consent. Exclusion criteria were subjects who used other facial skin rejuvenation therapy (injection of botulinum toxin, fillers, peels, microdermabrasion, antioxidants, skincare creams) and were uncooperative.

Prior to the study, the patients were educated and informed to sign informed consent voluntarily. All enrolled subjects were examined by Facial Analysis System JANUS-II ${ }^{\circledR}$ to evaluate wrinkles, pores, dark spots, and skin tone as baseline data. The subjects were then received topical maintenance treatment with $0.025 \%$ tretinoin and SPF 30 sunscreen. A twice 4 weekly evaluation was done using the same device. The obtained data were collected, input to a spreadsheet along with photo analysis of the face with
Janus-II, and analyzed. Ethical clearance was obtained before this research was conducted.

\section{RESULTS}

This study involved 30 research subjects who met the inclusion and exclusion criteria. The sampling was done consecutively. The basic characteristics of the research subjects are presented in Table 1 . The age range of the subjects was $46-55$ years old. There were 23 subjects (76.7\%) aged 46-55 years old, 5 subjects (17\%) aged $40-45$ years old, and 2 subjects $(6.6 \%)$ aged $56-65$ years old. The youngest subject was 40 years old, and the oldest subject was 64 years old.

Based on Glogau classification, which was evaluated shortly before long-term observations, 5 subjects (17\%) were classified into category II, and 25 subjects $(83 \%)$ were classified into category III. Fitzpatrick skin type showed results that 25 subjects $(83 \%)$ were categorized as type IV, and 5 subjects (17\%) were categorized as type V. The residence distribution of the research were $93 \%$ subjects resided in Surabaya, and 7\% subjects resided outside Surabaya. The occupational distribution was divided into indoor and outdoor. The collected data showed that $100 \%$ of participants had indoor works. The UV protection evaluation found that all subjects (100\%) used sunscreen, and 5 subjects (16.7\%) used an umbrella. The outdoor activity evaluation found that 25 subjects $(83 \%)$ rode motorbikes, and only 3 subjects $(10 \%)$ did gardening. The assessment of $0.025 \%$ tretinoin use confirmed that all subjects used the cream.

The statistical test resulted no significant differences between the evaluation 3, 4, and 5, and is presented in Table 2. The result indicated that there was a change in the degree of wrinkles during the evaluation, but it was not significant. As presented in Table 3, the statistical test results confirmed that there was a significant change in ultraviolet spots on the $5^{\text {th }}$ evaluation. No significant differences were found between the $3^{\text {rd }}$ and $4^{\text {th }}$ (p 0.053 ) evaluation. In Table 4, statistical test results showed that there was no significant difference between evaluation 3, 4, and 5 . This indicated that there were no significant changes between the UV spot values during the evaluation. In Table 5, the statistical test results showed that there was a significant difference between the evaluation 3, 4, and 5. This indicated that there were significant changes between the pore during evaluations. All study subjects completed the study evaluation within 3 months, and no side effects were found in all study subjects $(100 \%)$. 
Table 1. Demographic data of research subjects

\begin{tabular}{|c|c|}
\hline Variable & $\begin{array}{l}\text { Total }=30 \\
\mathrm{n}(\%)\end{array}$ \\
\hline \multicolumn{2}{|l|}{ Age } \\
\hline $40-45$ years old & $5(17)$ \\
\hline $46-55$ years old & $23(76.7)$ \\
\hline $56-65$ years old & $2(6.6)$ \\
\hline \multicolumn{2}{|l|}{ Glogau } \\
\hline Category II & $5(17)$ \\
\hline Category III & $25(83)$ \\
\hline \multicolumn{2}{|l|}{ Fitzpatrick skin } \\
\hline Type IV & $25(83)$ \\
\hline Type V & $5(17)$ \\
\hline \multicolumn{2}{|l|}{ Residence } \\
\hline Surabaya & $28(93)$ \\
\hline Outside Surabaya & $2(7)$ \\
\hline \multicolumn{2}{|l|}{ Occupation } \\
\hline Indoor & $30(100)$ \\
\hline Outdoor & $0(0)$ \\
\hline \multicolumn{2}{|l|}{ UV protection } \\
\hline Sunscreen & $30(100)$ \\
\hline Umbrella & $5(16.7)$ \\
\hline \multicolumn{2}{|l|}{ Outdoor activities } \\
\hline Traveling by motorcycle & $25(83 \%)$ \\
\hline Morning exercise & $20(67 \%)$ \\
\hline Gardening & $3(10 \%)$ \\
\hline Shop at the market & $10(33 \%)$ \\
\hline \multicolumn{2}{|c|}{ Using cream tretinoin $0.025 \%$ as maintenance therapy } \\
\hline Routine & $30(100)$ \\
\hline No & 0 \\
\hline
\end{tabular}

$\mathrm{UV}=$ Ultraviolet

Table 2. Results of wrinkle analysis on Janus evaluation

\begin{tabular}{clll}
\hline Janus 3 & Janus 4 & Janus 5 & $\mathrm{p}$ \\
\hline $7.0(4.0-23.0)$ & $6.5(3.0-13.0)$ & $6.0(0.0-12.0)$ & 0.095 \\
\hline
\end{tabular}

Friedman test $\mathrm{p}=0.095$

Table 3. Results of polarized spot analysis on Janus evaluations

\begin{tabular}{clll}
\hline Janus 3 & Janus 4 & Janus 5 & $\mathrm{p}$ \\
\hline $33.9 \pm 6.4$ & $33.03 \pm 7.66$ & $31.67 \pm 5.89$ & 0.002 \\
\hline
\end{tabular}

Annova test $\mathrm{p}=0.002$

Table 4. Results of ultraviolet analysis

\begin{tabular}{clll}
\hline Janus 3 & Janus 4 & Janus 5 & $\mathrm{p}$ \\
\hline $8.0(2.0-22.0)$ & $7.5(2.0-22.0)$ & $7.5(2.0-19.0)$ & 0.223 \\
\hline
\end{tabular}

Friedman test $\mathrm{p}=0.223$ 
Table 5. Results of pore analysis

\begin{tabular}{clll}
\hline Janus 3 & Janus 4 & Janus 5 & $\mathrm{p}$ \\
\hline $53.5(40.0-65.0)$ & $51.0(40.0-66.0)$ & $48.5(10.0-59.0)$ & $<0.0001$ \\
\hline Friedman test $\mathrm{p}<0.0001$ & & &
\end{tabular}

\section{DISCUSSION}

The age groups that experienced photoaging in the study were 46-55 years as many as 19 subjects $(63.3 \%)$. The youngest subject was 40 years old, and the oldest subject was 64 years old. This was similar to the research conducted by Lee and colleagues with 25 subjects aged 41-64 years old, and the average age was 51.6 years. ${ }^{9}$

Photoaging severity in this study was assessed using the Glogau scale. There were $5(18 \%)$ subjects categorized into Glogau II and 23 (83\%) subjects categorized into Glogau III. Glogau scale was used considering that its assessment criteria are the easiest and most applicable, providing effective measurements on treatment effectiveness. A study by El Kahky and colleagues on skin rejuvenation with mesenchymal stem cell treatment classified $70 \%$ of subjects into Glogau type II and 20\% into Glogau type III. Female patients with Glogau II and III photoaging usually have more apparent wrinkles and pigmentation changes such as lentigo and keratosis. They look for skin rejuvenation treatment to prevent worse clinical manifestation. ${ }^{10}$

UV radiation reaches more dermis layer on brighter than darker skin. Thus, individuals with low Fitzpatrick skin types tend to be more susceptible to photoaging. In Caucasian women, photoaging appears as clear deep wrinkles and skin dyspigmentation. In Asian women, the photoaging is mainly manifested on skin pigmentation accompanied by wrinkles especially under the eye. ${ }^{11}$

Skin type plays an important role in determining the visible effect of photoaging. Individuals with Fitzpatrick skin type I and II show signs of atrophic skin changes with fewer wrinkles and dysplastic premalignant changes such as actinic keratosis. In comparison, skin type III and IV display hypertrophic skin, wrinkles, rough skin, lentigines, and overall bronze appearance. ${ }^{12}$ In Asian persons, the primary manifestation of photodamage is a change in pigmentation rather than wrinkles. ${ }^{13}$ In this study, 25 subjects (83\%) had type IV skin, and 5 subjects (17\%) had type V skin. This was in accordance with the study by El Kahky and colleagues; the majority of patients who participated in the research had type IV (70\%) and type V $(30 \%)$ skin. $^{10}$

Indonesia is geographically located on the equator, and Surabaya is located at $7^{\circ} 9^{\prime}-7^{\circ} 21^{\prime}$ south latitude and $112^{\circ} 36^{\prime}$ '- $112^{\circ} 57^{\prime}$ east. Geographical location affects exposure to sunlight. Temperature Surabaya is around $33^{\circ} \mathrm{C}-34^{\circ} \mathrm{C}$, and the UV index is 911 ; it means that the ultraviolet light index is very strong. ${ }^{14}$

Before conducting this long-term evaluation study, all subjects had been given AMSC-MP after fractional $\mathrm{CO}_{2}$ laser as LADD. The treatment consisted of 3 sessions for 3 months and was evaluated 4 weeks after the last session. After the last session, the patients received follow-up evaluation using skin analyzer for 12 weeks to determine the long-term effects of topical AMSC-MP after fractional $\mathrm{CO}_{2}$ laser as LADD.

This study used an objective skin analyzer as an evaluation tool. There were four assessments measured in this study, namely wrinkles, dark spots (polarized and UV), pores, and skin tones. The normality test results showed that the assessment parameters were not normally distributed. This study showed that polarized spots and pores showed a long-term effect of topical AMSC-MP after fractional $\mathrm{CO}_{2}$ laser on photoaging. This was consistent with research conducted by Prakoeswa and his colleagues on AMSC-MP after micro-needling for antiaging treatment. There were significant clinical improvements from photoaging in the subjects. More improvements in clinical pores than wrinkles and spot were noticed. ${ }^{8}$

There were improvements in several parameters. This might be due to the topical effects of AMSC-MP that contain various growth factors, stimulating the proliferation and migration of dermal fibroblasts, epidermal keratinocytes, and increasing collagen synthesis from fibroblasts and fractional $\mathrm{CO}_{2}$ laser as LADD. The use of $0.025 \%$ tretinoin and SPF 30 sunscreen aimed to provide better UV protection. To date, there is on long-term effects of AMSC-MP on the skin tissue.

The UV radiation can cause the formation of wrinkles through reduced stress strength and elasticity and can cause degradation of structural components that support the dermal extracellular matrix. The UV radiation can also increase reactive oxygen species (ROS), increasing activator protein 1(AP-1) and nuclear factor-k $\beta$ (NF-kB), reducing transforming growth factor $\beta$ (TGF- $\beta$ ); therefore, decreasing collagen production and promoting collagen damage.

Damaged tissue and aging cells are restored with continuous skin renewal. Treatment of tissue homeostasis depends on stem cell activity, and it decreases over time or through constant UVB 
exposure. The reduced ability to repair trauma is the hallmark of skin aging. AMSC-MP also have a rejuvenating effect from the secretion factor through the activation of dermal fibroblasts and inhibit melanogenesis. Extracellular matrix (ECM) is an important component, and it interacts with stem cells through complex macromolecular networks that regulate proliferation and differentiation. ECM maintains stem cells in the right location and provides the rigidity needed for stem cell survival. Changes in the structure and composition of ECM, especially collagen fibers, during the aging process reduce tissue stiffness and elasticity. A higher dose of fractional $\mathrm{CO} 2$ laser is needed than the dose needed to induce reepithelialization, and the ideal number of treatment sessions for optimal fractional ablative laser rejuvenation requires further research and longer follow-up. ${ }^{15}$

Melanin is a pigment in the epidermis, and it gives color to the skin. Exposure to UV light can increase the activity of the tyrosinase enzyme, promoting tyrosine change into dopamine (DOPA) and DOPA-quinone. This will cause melanocytes to produce more melanin. Melanin will be distributed through melanosomes, thus giving color to the keratinocyte layer. Black spots occur as a result of melanin accumulation due to continuous sun exposure. Research conducted by Seo and colleagues showed that stem cells have a whitening effect through inhibition of melanin synthesis and tyrosinase activity, and they can reduce the expression of melanogenic enzymes. ${ }^{16}$

During AMSC-MP and laser fractional $\mathrm{CO}_{2}$ treatment, polarized spots worsen, but long-term evaluation found that the spots improved significantly. It is presumably because the AMSC-MP reduce the expression of melatonin receptors $1 \mathrm{~A}$ and tyrosinase, thereby affecting the outcome spots black because of UVB. ${ }^{15}$

UVB irradiation is known to stimulate melanogenesis and tyrosinase through bone morphogenic protein-4 (BMP4) and its receptors BMP$1 \mathrm{~b}$ in the skin. BMP4 significantly inhibits melanogenesis by decreasing the regulation of tyrosinase expression and activity below the initial level. The mechanism of the BMP4 effect on tyrosinase and finally on melanogenesis involves a moderate reduction in the rate of transcription of tyrosinase and stability of messenger ribonucleic acid (mRNA). UVB irradiation stimulates melanogenesis by decreasing the regulation of BMP-1B receptor mRNA in melanocytes. It was found that UVB irradiation increased the regulation of melatonin $1 \mathrm{~A}$ receptor expression and tyrosinase, adipose-derived stem cell (ADSC) transplantation reduced the expression of melatonin
$1 \mathrm{~A}$, and tyrosinase receptors in contrast with the results observed for BMP4. ADSC reverses typical photoaging signs by remodeling skin cell niches through modulation of the BMP4 pathway and transdifferentiation into skin stem cells. This discovery provides theoretical support for the treatment of skin photoaging with ADSC transplants. ${ }^{17}$

Research conducted by Zhou and his colleagues in China on 9 research subjects given conditioned medium from ADSC after fractional $\mathrm{CO}_{2}$ laser showed good results in skin rejuvenation characterized by improved histological assessment and biophysical analysis such as erythema index, melanin index, transepidermal water loss (TEWL), hydration, skin roughness. ${ }^{18}$

During the treatment, UV spots worsened. In post-treatment evaluations, the UV repair results were not significant. This was allegedly due to external factors that affect the black spots (UV), thus affecting the black spot. In addition, UV spots are less visible than polarized spots

Skin tone observed to be significantly worsened during treatment and also 3 months after. Skin tone is influenced by a variety of factors, including skin types, excessive workload, stress, melanin production, sunburn, keratin, skin problems, and so on. Each subject has different levels of stress, duration of sun exposure, melanin production, and skin problems. Uniforming these factors is very difficult, considering that stress is an internal factor that cannot be measured quantitatively. In stress conditions, the skin received less blood flow, making it become pale. Another factor is melanin production, which is affected by the length of sun exposure and skin type. ${ }^{15}$

During AMSC-MP and fractional $\mathrm{CO}_{2}$ laser treatment, the skin pores were deteriorated significantly, while in post-treatment evaluations, it shows significant improvement. Enlarged pores are influenced by various factors, one of which is skin damage due to photodamage. Photodamage can cause a decrease in the expression of glycoprotein related to microfibril-1 (MAGP-1), which is an important component in the assembly of elastic fibers, thereby affecting the elasticity of the skin in the dermis area and pores. AMSC-MP has a variety of growth factors that are proven to increase collagen synthesis. Research carried out by Lee, and his colleagues showed an improvement in the degree of wrinkles and skin roughness in the application of stem cell metabolite products. ${ }^{9}$

No side effects were observed in the study subjects for 3 months of evaluation, in the form of persistent erythema, itching, post-inflammatory hyperpigmentation, hypopigmentation, scarring, or 
infection. This was allegedly due to using metabolite products from stem cells without these cells. The use of this metabolite product is easier and safer because it is acellular, so it is easier to control and can prevent tissue rejection reactions in recipients than products that use cells when applied to wounds. ${ }^{19}$ The fractional $\mathrm{CO}_{2}$ laser treatment performed in this study was LADD technique with low energy so that there was no effect of scarring. After facial laser treatment, the patients received wound care using gentamicin ointment to avoid infection.

This research concluded that there were clinical improvements on wrinkles, polarized spots (polarized), UV spots, and pores in photoaging patients 12 weeks after completed 3 sessions of 4-weekly AMSC-MP and fractional $\mathrm{CO}_{2}$ laser. There were no side effects observed.

\section{REFERENCES}

1. Fisher GJ, Varani J, J Voorhees. Looking older: fibroblasts collapse and therapeutic implications. Arch Dermatol 2008; 144(5):666-72.

2. May X, Wang L. Ablative fractional carbon dioxide laser combined with intense pulsed light for the treatment of photoaging skin in Chinese population. A split-face study. Mei and Wang Medicine 2018; 97:3.

3. Goswami P. Ageing and its effect on body-self image, mood and self esteem of middle age women and older women. IOSR Journal of Humanities and Social Science 2013; 18(5): 6373.

4. Saluja S, Sabrina GA. Holistic approach to antiaging as an adjunct to antiaging procedures: a review of the literature. Dermatol Surg 2017; 43: 475-84.

5. Kim W, Park B, Park S, Kim H, Sung J. Antiwrinkle effect of adipose-derived stem cells: activation of dermal fibroblasts by secretory factors. J Dermatol Sci 2009; 53 (2):96-102.

6. Panich U, Sittithumcharee G, Rathviboon N. Ultraviolet radiation-induced skin aging: The role of DNA damage and oxidative stress in epidermal stem cell damage mediated skin Aging. Stem Cells Int 2016: 73(7): 1-14.

7. Ibrahim O, Wenande E, Hogan S, Arndt KA, Haedersal M, Dover JS . Challenges to laserassisted drug delivery: applying theory to clinical practice. Lasers Surg Med 2017; 20: 1-8.

8. Prakoeswa CRS, Pratiwi FD, Herwanto N, Citrashanty I, Indramaya D et al. The effects of amniotic membrane stem cell-conditioned medium on photoaging. Journal of Dermatological Treatment 2018; 1-15.
9. Lee HJ, Lee EG, Kan G S, Sung JH, Chung HM, Kim DH. Efficacy of microneedling plus human stem cell conditioned medium for skin rejuvenation: randomized, controlled, blinded split-face study. Ann Dermatol 2014; (26): 58490.

10. El-Kahky, H. El-Hefnawy, Aatia AH, Mohammed M, Ismai 1. An analysis of the rejuvenative effect of mesenchymal stem cells on aged skin: comparative evaluation of two types; bone marrow-derived and adipose-derived stem cells. J Stem Cells Gens 2017; 1(1): 1-7.

11. Ahmad Z, Damayanti. Skin Aging: Pathophysiology and Clinical Managing. Berkala Ilmu Kesehatan Kulit dan Kelamin 2018; 30(3): 208-15.

12. Poon F, Kang S, Chien A. Mechanisms and treatments of photoaging. Photodermatology Photoimmunology Photomedicine 2015; 31: 6574.

13. Yan S, Xu F, Yang C, Li F, Kan G S, Sung JH et al. Demographic differences in sun protection beliefs and behaviors: a community-based study in shanghai, China. Int $\mathrm{J}$ Environ Res Public Health 2015; 12: 3232-45.

14. RJPD. Surabaya City Medium Term Development Plan 2016-2021. p-1. 2016. Accessed $29 \quad$ October $2016 \quad 201$. http://surabaya.go.id/uploads/attachments/2016/1 1/16408/bab_2.pdf

15. Ichihashi $M$. Photoaging of the skin. Anti Aging Med 2009; 6 (6): 46-59.

16. Seo KY, Kim D H, Lee SE, Yoon MS, Lee HJ. Skin rejuvenation by microneedle fractional radiofrequency and a human stem cell conditioned medium in Asian skin: a randomized controlled investigator blinded split-face study. J Cosmet Laser Ther 2013; 15(1):25-33.

17. Gong $\mathrm{M}$, Zhang $\mathrm{P}$, Li C, Ma X, Yang D. Protective mechanism of adipose-derived stem cells in remodeling of the skin stem cell niche during photoaging. Cell Physiol Biochem 2018; 51: 2456-71.

18. Zhou B, Zhang T, Jameel AA, Xu Y, Xu Y, Guo $\mathrm{S}$, et al . The efficacy of conditioned media of adipose derived stem cells combined with ablative carbon dioxide fractional resurfacing for atrophic acne scars and skin rejuvenation. J Cosm Laser Ther 2016; 1(2): 1-31.

19. Tamam AK, Kerpedjieva SS. Acceleration of wound healing by multiple growth factors and cytokines secreted from multipotential stromal cells / mesenchymal stem cells. Adv Wound Care 2012; 1(4): 177-82. 